\title{
BreastCore
}

\section{Prophylactic Surgery: For Whom, When and How?}

\author{
Christine Mau Michael Untch
}

Interdisziplinäres Brustzentrum, Helios-Klinikum Berlin-Buch, Berlin, Germany

\section{Keywords}

Hereditary breast and ovarian cancer - Risk reduction . Surgery

\section{Summary}

Risk-reducing surgery has proved to be a reasonable procedure in healthy women with a definitely elevated risk of developing cancer. Here we consider the elevated risk of breast and ovarian cancer. There is a clear indication for such surgery in healthy women with a pathogenic BRCA1/2 mutation. For these patients, a risk-reducing bilateral mastectomy leads to a verifiable reduction in mortality from breast cancer, particularly for young patients. In most cases, surgery is combined with breast reconstruction. The pros and cons of surgical treatment and the different surgical techniques have to be explained to and carefully considered with the patient. As yet, no unequivocal data for the benefits of intensifying early detection have been ascertained with respect to mortality from breast carcinoma. In index patients with a BRCA mutation, the surgical treatment should depend on the prognosis of the primary disease. A lower age at onset and a better prognosis of the primary disease make a contralateral mastectomy (CPM) more reasonable. In the case of BRCA mutation-related cancer, a reduction of mortality through CPM has been proven. A risk-reducing adnexectomy is basically recommended for BRCA mutation carriers. Healthy premenopausal women need a subsequent hormone replacement therapy. The prognosis of the patients is dominated by the ovarian carcinoma. This can be prevented by risk-reducing salpingo-oophorectomy in 95\% of the cases.

(C) 2017 S. Karger GmbH, Freiburg

\section{Introduction}

With approximately 69,500 new cases and 17,000 deaths per year, breast cancer is the most frequent type of cancer in women. For the years 2011/2012, the Robert Koch Institute reported that the relative survival rates range from $83 \%$ to $89 \%$ after 5 years and from $79 \%$ to $83 \%$ after 10 years [1]. Most patients initially recover from breast cancer. Cancer registries for a long-term follow-up of more than 10 years are only occasionally available. Studies on adjuvant anti-estrogen therapy revealed that there is a risk of metastasis even after 15 or 20 years. According to the Federal Office of Statistics, the average life expectancy for women is 85 years. Hence, for patients developing cancer at a young age, therapy planning also has to take the long lifespan into consideration. Patients with hereditary disposition contract the disease at a considerably younger age; the average age of onset in BRCA1-positive patients is 44 years and in BRCA2-positive patients 47 years [2-4]. The mean age of onset for sporadic breast cancer is 64 years, whereby every fourth woman affected is younger than 55 and every tenth younger than 45 years.

There is a distinction between primary and secondary prevention. Primary prevention represents the actual prevention of the disease, while secondary prevention is synonymous with intensified early detection. Tertiary prevention stands for life-long aftercare.

Table 1 gives an overview of highly and moderately penetrant genes causing a predisposition for breast cancer, and the measures that can be offered to people seeking advice (healthy mutation carriers). As a rule, intensified early detection routines comprise a breast sonography every 6 months and a magnetic resonance (MR) mammography once a year from the age of 25 onwards as well as a mammography every 1-2 years from the age of 40 onwards [5]. Evidence of a family history of breast carcinoma or other (relevant) types of cancer, provided inclusion criteria [6] are fulfilled, should prompt a genetic analysis. In this context, the family tree is frequently not a sufficiently sensitive instrument for proving a hereditary cause for a malignant disease. In some cases, genetic testing is only indicated at the onset of disease. An overview for examination of the hereditary causes of breast cancer is given in table 2 . 
Table 1. Overview of hereditary dispositions, risks of disease and measures

\begin{tabular}{|c|c|c|c|c|}
\hline Gene & Lifetime risk for breast cancer & [Ref.] & $\begin{array}{l}\text { Intensified } \\
\text { early } \\
\text { detection }\end{array}$ & Risk-reducing mastectomy \\
\hline BRCA1 & $65 \%(44-78 \%)$ & {$[18-21]$} & yes & reasonable option \\
\hline BRCA2 & $55 \%(51-70 \%)$ & {$[18-21]$} & yes & reasonable option \\
\hline PALB2 & $\begin{array}{l}\text { risk of breast carcinoma increased } \\
3-5 \text { times, depending on family } \\
\text { anamnesis }\end{array}$ & {$[9,10]$} & yes & case-by-case decision \\
\hline CHECK2 & $\begin{array}{l}\text { risk of breast carcinoma increased } \\
2-5 \text { times, depending on family } \\
\text { anamnesis and mutation }\end{array}$ & {$[6,7]$} & yes & case-by-case decision \\
\hline CDH1 & lobular breast carcinomas $30-50 \%$ & [2] & yes & case-by-case decision \\
\hline TP53 & approx. $50 \%$ & [1] & yes & case-by-case decision \\
\hline PTEN & $80 \%$ & [3] & yes & no \\
\hline STK11 & $30-50 \%$ & [4] & yes & no \\
\hline ATM & $\begin{array}{l}\text { risk of breast carcinoma increased } \\
2-4 \text { times, depending on family } \\
\text { anamnesis }\end{array}$ & {$[11,12]$} & yes & no \\
\hline RAD51 C & $\begin{array}{l}\text { risk of ovarian carcinoma possibly } \\
20-40 \% \text {, risk of breast carcinoma } \\
\text { uncertain, depending on family } \\
\text { anamnesis }\end{array}$ & {$[6,13]$} & yes & $\begin{array}{l}\text { no } \\
\text { recommendation of prophylactic } \\
\text { adnexectomy }\end{array}$ \\
\hline RAD51 D & $\begin{array}{l}\text { risk of ovarian cancer uncertain, } \\
\text { possibly } 10 \% \text {, risk of breast c } \\
\text { arcinoma uncertain }\end{array}$ & {$[13,14]$} & yes & $\begin{array}{l}\text { no } \\
\text { prophylactic adnexectomy } \\
\text { possible in case of further } \\
\text { ovarian carcinomas in the family }\end{array}$ \\
\hline NBN & $\begin{array}{l}\text { risk of breast carcinoma increased } \\
2-3 \text { times, depending on family } \\
\text { anamnesis }\end{array}$ & {$[18,19]$} & yes & no \\
\hline $\begin{array}{l}\text { LYNCH syndrome } \\
\text { (MLH1, MSH2, } \\
\text { MSH6, PMS2) }\end{array}$ & $\begin{array}{l}\text { colorectal carcinomas } 80 \% \text {, } \\
\text { endometrium carcinomas } 20 \text { - } \\
60 \% \text {, ovarian carcinomas } 10 \%\end{array}$ & {$[15]$} & & no \\
\hline
\end{tabular}

Table 2. Description of indications of testing for hereditary dispositions with fulfilled inclusion criteria

\begin{tabular}{ll}
\hline Index patients & People seeking advice \\
\hline $\begin{array}{c}\text { In primary disease situation of } \\
\text { breast carcinoma under primary } \\
\text { chemotherapy }\end{array}$ & $\begin{array}{l}\text { healthy relatives from informative } \\
\text { families (with proven pathogenic } \\
\text { mutation) }\end{array}$ \\
$\begin{array}{c}\text { In primary disease situation of } \\
\text { breast carcinoma at very early }\end{array}$ & $\begin{array}{l}\text { probability and deceased index } \\
\text { patients }\end{array}$ \\
age of onset & $\begin{array}{l}\text { caveat: appropriate time to consider } \\
\text { after primary consultation }\end{array}$ \\
carcinarian carcinoma or breast & non-directive consultation on \\
In disease-free interval after primary & consequences of testing \\
onset &
\end{tabular}

\section{Primary Prevention of Breast Cancer}

For healthy BRCA1/2 mutation carriers, risk-reducing mastectomy (RRM) is a very reasonable option (fig. 1). This, however, does not apply to all other highly and moderately penetrant genes, for which at present insufficient data are available. In the case of highly penetrant genes and/or highly burdened family anamnesis, risks and benefits have to be carefully considered jointly with the patient seeking advice on a case-by-case basis. Prior to performing RRM, it has to be clarified whether the costs will be covered by the health insurance.

RRM prevents the development of breast cancer in approximately $95-98 \%$ of all patients. Comprehensive patient information about the pros and cons of risk-reducing surgery is essential. The consultation has to be all-encompassing, if need be, several times, and sufficient time for consideration must be given. Moreover, comprehensive information on the different possibilities of breast reconstruction is mandatory, as is advice regarding possible complications and follow-on surgery. Under ideal conditions, patient information takes place in the presence of the patient's partner. The sensory loss of skin and areolae should be explained. Advice on the residual risk of primary breast carcinoma should be included. To assess the possibly of remaining residual parenchyma, a breast MR tomography (MRT) is carried out generally 1 year after surgery. If the MRT does not reveal residual parenchyma, a breast sonography at annual intervals is sufficient for aftercare provided palpation findings are normal. 

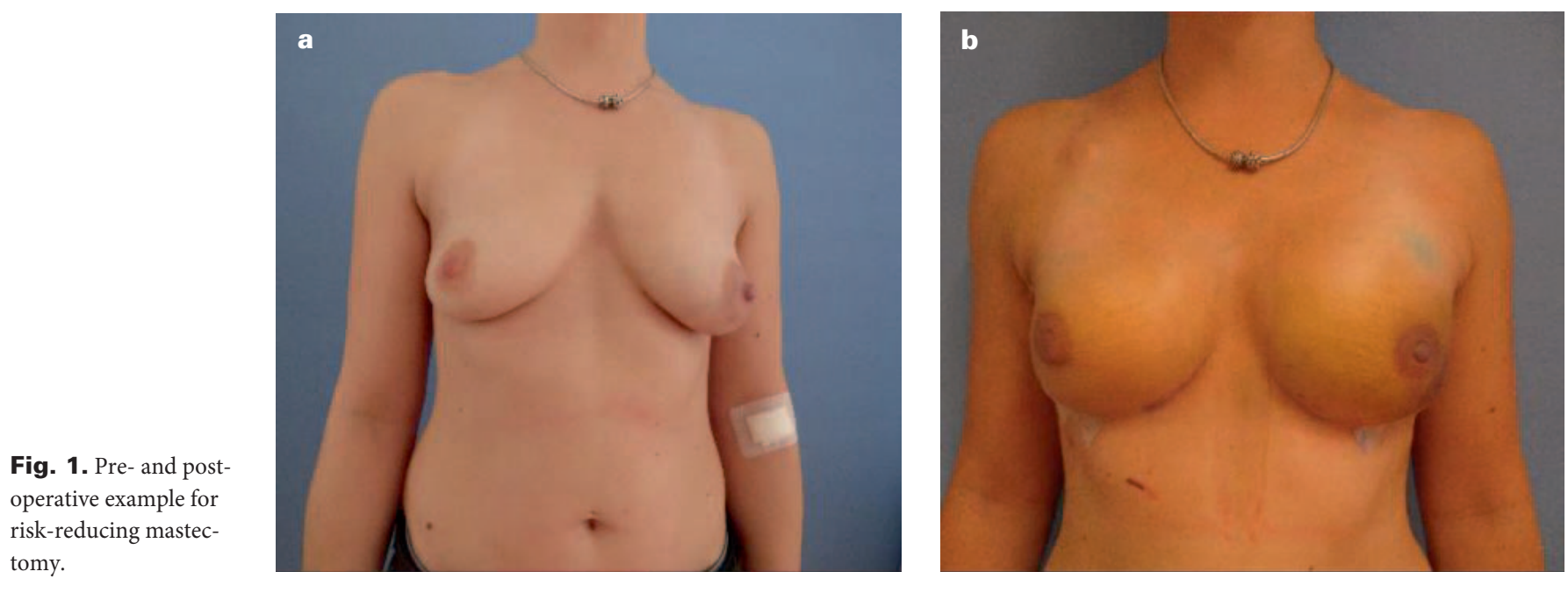

The skin and areola-conserving mastectomy, in comparison to a modified radical mastectomy, has proven to be safe also in BRCA carriers; the local recurrence rate after $5-7$ years is $3.5-5.5 \%$ [713]. The patients should be offered an immediate reconstruction that can be performed using either silicone implants or the patient's own tissue depending on age, habits, comorbidities, and patient's wishes (see below for technical details).

The 2010 update of the Cochrane Database analysis on RRM [6] covers 39 surveillance studies with a total of 7,384 women after bilateral prophylactic mastectomy. There are no randomized studies, which would be out of the question for ethical reasons. The analysis showed a reduction of both the incidence of breast cancer and breast cancer-specific mortality, particularly in BRCA1/2 carriers. The studies also point towards a measurable reduction of stress and fear levels after RRM. Generally, the patients were highly satisfied with the cosmetic results; however, re-surgery is necessary after RRM in up to $49 \%$ of the patients. RRM thus represents a suitable measure for preventing the development of breast cancer in healthy BRCA-positive women. An alternative approach (see above) is possible.

\section{Secondary Prevention of Breast Cancer}

In this section, possible treatment options for patients already suffering from breast cancer (index patients) are discussed. Basically, there is a distinction between the approaches in the primary disease situation with evidence for mutation, in the disease-free interval, and in the therapy if ipsilateral or contralateral local recurrence arises. The approach in case of metastasis is not considered.

Is breast-conserving surgery advisable for primary disease in BRCA mutation carriers? Should a bilateral mastectomy be performed in any case? To answer these questions it is necessary to consider the age of the woman concerned, the type of existing mutation and the prognosis of the primary disease. The earlier the age of onset, the higher is the potential benefit of bilateral mastectomy. The rate of ipsilateral and contralateral recurrences is higher for BRCA1 mutations than for BRCA2 mutations. In patients in whom the primary disease has a bad prognosis, the lymph nodes have been severely affected, or there has been a bad response to primary chemotherapy, the risk of metastasis is very high, and the woman should be advised against bilateral mastectomy. If metastasis has already occurred, this approach is out of the question.

A patient with newly diagnosed breast carcinoma who fulfills the criteria should be offered gene testing to determine whether there is an hereditary cause as that has an influence on therapy planning. Some patient wish to receive a different surgical treatment than that originally envisaged.

The circumstances are different for a pathogenic mutation is newly diagnosed during the disease-free interval after primary disease. The prognosis, the age, and the time since first onset have to be taken into consideration and discussed with the patient. Before performing bilateral mastectomy, it is advisable to exclude metastasis. Rhiem et al. [14] have published tables relating to the probability of contracting the disease contralaterally, depending on the age at first onset and the time lag. These can be used by both physician and patient to assess the risk of contracting contralateral disease within the next 5 or 10 years.

If intramammary or contralateral local recurrence occurs, the approach depends on age, mutation, prognosis, and comorbidities. When chemotherapy is advisable, it should be administered, preferably as neoadjuvant or primary systemic therapy since the outcome will affect prognosis.

\section{Ipsilateral Recurrence}

Biglia et al. [15] give a good summary as to the rate of ipsilateral-breast recurrence (IBR) after breast-conserving therapy in cases with BRCA mutation. A recently published meta-analysis [16] from 6 cohort studies and 4 case-control studies of 526 BRCA carriers and 2,320 control patients showed no significant increase of the local recurrence risk after breast-preserving therapy and radiation $(17.3 \%$ vs. $11 \%$, risk ratio (RR) $1.45,95 \%$ confidence interval (CI) $0.98-2.24, \mathrm{p}=0.07$ ). When classifying the studies according to the duration of aftercare, in a follow-up over 7 years ( 5 studies, 1,634 patients), a significantly higher rate of IBRs was shown (15.9\% vs. $7 \%$, RR 1.51, 95\% CI 1.15-1.98, p < 0.003) for breast- 
conserving therapy compared to mastectomy. This could be explained by higher incidence of de novo carcinomas in mutation carriers. The overall survival (OS) showed no difference.

Only 2 studies (893 patients) presented separate data on true recurrences and new primary cancer $[17,18]$. The median follow-ups of these studies were 4.5 and 3.4 years respectively. They showed no significant increase in true recurrences (RR 1.37, 95\% CI 0.44$4.21, \mathrm{p}=0.59)$ but a trend for higher new primary cancers (RR $2.07,95 \%$ CI $0.99-4.36, \mathrm{p}=0.05)$ in BRCA-mutation carriers versus non-carriers. Only 1 study on OS [17] showed a significant difference between the 2 groups with $94 \%$ OS for the prophylactic mastectomy group versus $77 \%$ for the non-prophylactic mastectomy group $(\mathrm{p}=0.03)$. However, when adjusted for other factors such as prophylactic oophorectomy, women in the first group did not show a significantly better survival than those in the second group (hazard ratio (HR) $0.35, \mathrm{p}=0.14$ ). No benefit of Tamoxifen in fighting IBC was seen. Affected lymph nodes ipsilaterally are a negative predictor for IBR [18].

\section{Contralateral Recurrence}

Administering adjuvant chemotherapy and performing an adnexectomy had a protective effect against contralateral-breast recurrence (CBR) (RR 0.52, 95\% CI 0.37-0.74). Onset of disease at an earlier age $(<50)$ was associated with a higher risk of CBR [19]. Administering of adjuvant systemic treatment (including antibody treatment and hormones) and adnexectomy were associated with a reduction of the risk of CBR by $50 \%$.

A prospective analysis from the Netherlands showed a survival benefit for contralateral mastectomy [20]. In this analysis, 583 BRCA-associated primary breast cancer patients diagnosed between 1980 and 2011 were selected from a multicenter cohort. Over time, 242 patients (42\%) underwent contralateral RRM (CRRM) and 341 patients (58\%) remained under surveillance. CBR was detected in 4 patients (2\%) after CRRM, and in 64 patients of the surveillance group (19\%). The majority of the CBRs had a favorable tumor stage, with a Tis/T1 classification in $87 \%$, and nodenegative disease in $79 \%$ of the patients. However, $73 \%$ of the tumors were triple negative. CBR was diagnosed in $13 \%$ of the BRCA1 patients and in $8 \%$ of the BRCA2 patients $(\mathrm{p}=0.122)$. Median follow-up after CBR diagnosis was 5.2 years (range 0.115.5 years). 16 of the CBR patients (24\%) died during follow-up, all in the surveillance group. The mortality was lower in the CRRM group (21.6 vs. 9.6 per 1000 person-years of observation); the Cox analysis yielded an HR of 0.49 (95\% CI, 0.29-0.82; adjusted for risk-reducing salpingo-oophorectomy (RRSO)) [20]. However, a bias is assumed for this study since in the CRRM group a higher proportion of women underwent adjuvant chemotherapy, which might have an impact on the difference in survival rates.

In 2014, Metcalfe et al. [21] published a major study with BRCA-positive patients and a long follow-up. 390 women with a family history of stage I or II breast cancer who were carriers of BRCA 1 and BRCA 2 mutations and initially treated with unilateral or bilateral mastectomy. 181 patients had mastectomy of the contralateral breast. Patients were followed for up to 20 years from di- agnosis. In a multivariable analysis, controlling for age at diagnosis, year of diagnosis, treatment, and other prognostic features, contralateral mastectomy was associated with a $48 \%$ reduction in death from breast cancer (HR 0.52, 95\% CI 0.29-0.93; p = 0.03).

In summary, it can be stated that BRCA mutation carriers (BRCA1 > BRCA2) with early onset of the disease and good prognosis of their primary disease will most likely benefit from a bilateral RRM, and that in healthy mutation carriers this can mostly prevent the disease.

\section{RRM Technique}

There is a distinction between primary 1-stage and secondary 2-stage reconstruction, as well as between autologous and heterologous breast reconstruction; moreover, there are various 1-stage and 2-stage procedures for skin tightening. Essentially, a removal of the mammary gland body with resection of the axillary tail in terms of a complete glandectomy has to be postulated. In this context, the term 'conservative mastectomy' was developed and first used by Nava et al. [22] in 2009. It outlined the need for preservation of mammary appearance, biomechanical balance, adequate volume restoration and symmetrical scarring in oncoplastic surgery.

\section{Primary Prevention}

If the patient wishes a breast reconstruction, the skin and areolapreserving mastectomy is preferred. Removal of the areola is not a safety requirement. In young, slim patients with less ptotic breasts, immediate reconstruction using a subpectoral implant, with a mesh if the circumstances require, can lead to good results. A too-slim patient with minor subcutaneous tissue might suffer from complications due to reduced perfusion of skin or areola; moreover, the thin soft tissue coverage occasionally leads to insufficient cosmetic results. In this case, the surgeon has to balance and minimize further risks such as diabetes mellitus, nicotine abuse, or nipple piercings. In the case of ptotic breasts, a 2-stage approach with expansion prior to the nipple-sparing mastectomy (NSM) can be reasonable for a better outcome if the patient accepts to undergo 2 surgeries [23]. For patients with macromastia, a 2-stage approach with reduction and tightening of the breast envelope and subsequent NSM can be reasonable [24]. The loss in sensibility of skin and areola is mostly tolerated by patients from a subjective point of view [25]. For patients wishing a reconstruction with autologous tissue, a 2-stage approach with primary NSM, subcutaneous implant and conversion to autologous tissue is an option. This is particularly recommended for patients suffering from breast carcinoma.

\section{Secondary Prevention (Index Patients)}

If a patient needs radiation to the chest wall and/or lymphatic drainage during the primary stage of disease, an immediate breast reconstruction is significantly more prone to complications. Wound healing disorders should not be allowed to impair the application of radiation therapy in the appropriate therapeutic window since this would reduce oncological safety. 
The different techniques of immediate reconstruction using silicone implants for preserving the breast envelope and subsequent conversion to autologous reconstruction (immediate implant delayed autologous) enable the preservation of the breast envelope if a sufficient distance between the tumor and the surgical margin can be achieved [26]. Various concepts are advocated: (1) subcutaneous implant, radiation and conversion to own tissue after an interval; (2) temporary preservation of the skin for autologous tissue transfer until receipt of the histological findings of the surgical margins (buried flap); (3) radiation after reconstruction with autologous tissue; and (4) neoadjuvant radiation of the original mammary gland body prior to NSM (IDEAL concept) [27].

A final determination of the optimal approach can only be made on a case-by-case basis and depends on disease situation, habits and patient's wish. If postoperative radiation is indicated, a possibility must be found how to apply it and nonetheless achieve a satisfying cosmetic result.

\section{Quality of Life}

Better cosmetic results and higher patient satisfaction are achieved using NSM. In 2000, Frost et al. [28] published a descriptive study of all still-living women $(n=609)$ who had a family history of breast cancer and had elected to undergo bilateral prophylactic mastectomy at a large, tertiary US health care clinic between 1960 and 1993. 94\% ( $\mathrm{n}=572)$ of these women completed a study questionnaire. The mean time from prophylactic mastectomy to last follow-up was 14.5 years. Most women (70\%) were satisfied with the procedure; $11 \%$ were neutral; and $19 \%$ were dissatisfied. Among the psychological and social variables, the most striking finding was that $74 \%$ reported a diminished level of emotional concern about developing breast cancer. The majority of women reported no change/favorable effects in levels of emotional stability $(68 \% / 23 \%)$, level of stress $(58 \% / 28 \%)$, self-esteem $(69 \% / 13 \%)$, sexual relationships $(73 \% / 4 \%)$, and feelings of femininity $(67 \% / 8 \%)$. $48 \%$ reported no change in their level of satisfaction with body appearance; $16 \%$ reported favorable effects. However, $9 \%, 14 \%, 18 \%$, $23 \%, 25 \%$, and $36 \%$ reported negative effects in these 6 variables, respectively.

\section{Cost Efficiency}

Zendejas et al. [29] carried out an interesting calculation. Using a Markov model, they simulated patients with breast cancer from mastectomy to death. Model parameters were gathered from published literature or national databases. Base-case analysis focused on patients with average-risk breast cancer, 45 years of age at treatment. Outcomes were valued in quality-adjusted life years (QALYs). Patients' age, risk level of breast cancer, and quality of life (QOL) were varied to assess their impact on results.

Mean costs of treatment for women age 45 years are comparable: $\$ 36,594$ for the contralateral mastectomy (CPM) and $\$ 35,182$ for surveillance. CPM provides 21.22 mean QALYs compared with 20.93 for surveillance, resulting in an incremental cost-effectiveness ratio (ICER) of $\$ 4,869 / \mathrm{QALY}$ gained for CPM. To prevent 1 CBR, 6 CPMs would be needed. CPM is no longer cost effective for patients older than 70 years (ICER \$62,750/QALY). For BRCApositive patients, CPM is clearly cost effective, providing more QALYs, while being less costly. In non-BRCA patients, cost effectiveness of CPM is highly dependent on assumptions regarding QOL for CPM versus surveillance strategy.

If a CRRM has to be performed 6 times to prevent 1 contralateral breast carcinoma, this represents a favorable ratio (number needed to treat 1:6). This is confirmed by German data [30,31]. From the German Statutory Health Insurance (SHI) perspective, undergoing bilateral mastectomy plus immediate bilateral salpinooophorectomy (BSO) should be recommended to BRCA1 or $2 \mathrm{mu}$ tation carriers due to its favorable comparative cost effectiveness.

\section{RRSO for Preventing Ovarian Carcinoma - For Whom?}

For BRCA1/2 carriers seeking advice, if family planning is finished and no concurrent (co)morbidities are present, RRSO always should performed. Risk-reducing surgery could potentially prevent at least $90 \%$ of epithelial ovarian cancers [32]. For premenopausal healthy women hormone replacement therapy is highly recommended [33]. For carriers of RAD51 C and D, RRSO is always recommended. For other core genes, no RRSO should be performed.

RRSO should be performed for BRCA1/2 index patients of younger age, with good prognosis and a sufficient time lap to primary onset of breast cancer. The prognosis of the breast cancer will not be worsened by RRSO.

\section{Conclusions}

Breast cancer risk reduction is important for known BRCA $1 / 2$ carriers. The biggest risk reduction can be achieved by performing risk-reducing surgery in healthy women. The decision is more difficult in index patients. The younger the patient and the better the prognosis of the disease, the higher is the benefit of risk-reduction surgery, e.g. for a BRCA1/2 carrier with triple-negative breast cancer and complete pathological remission after neoadjuvant chemotherapy. Therefore, the need for BRCA testing should be verified during neoadjuvant therapy. An unsolved problem is the assessment of the prognosis of an index patient. Randomized trials are impossible for ethical reasons. So the discussion of pros and cons of risk-reducing surgery is the fairest solution for the individual patient. Further data collection is necessary.

\section{Disclosure Statement}

The authors declare no conflicts of interest. 


\section{References}

1 RKI Krebs in Deutschland 2011/2012 www.rki.de

2 Hartmann LC, Lindor NM: The role of risk-reducing surgery in hereditary breast and ovarian cancer. N Engl J Med 2016;374:454-468.

3 Mavaddat N, Peock S, Frost D, et al.: Cancer risks for BRCA1 and BRCA2 mutation carriers: Results from prospective analysis of EMBRACE. J Natl Cancer Inst 2013; 105:812-822.

4 Kast K, Rhiem K, Wappenschmidt B, et al.: Prevalence of BRCA1/2 germline mutations in 21401 families with breast and ovarian cancer. J Med Genet 2016;53: 465-471.

5 http://www.konsortium-familiaerer-brustkrebs.de/ konsensusempfehlung.

6 Lostumbo L, Carbine NE, Wallace J: Prophylactic mastectomy for the prevention of breast cancer. Cochrane Database Syst Rev 2010;(11):CD002748.

7 Carlson GW, Styblo TM, Lyles RH, et al.: Local recurrence after skin-sparing mastectomy: Tumor biology or surgical conservatism? Ann Surg Oncol 2003;10 108-112.

8 Missana MC, Laurent I, Germain M, et al.: Long-term oncological results after 400 skin-sparing mastectomies. J Visc Surg 2013;150:313-320.

9 Reynolds C, Davidson JA, Lindor NM, et al.: Prophylactic and therapeutic mastectomy in BRCA mutation carriers: Can the nipple be preserved? Ann Surg Oncol 2011;18:3102-3109.

10 Tung N: Management of women with BRCA mutations: A 41-year-old woman with a BRCA mutation and a recent history of breast cancer. JAMA 2011;305 2211-2220.

11 Meijers-Heijboer H, van Geel B, van Putten WL, et al. Breast cancer after prophylactic bilateral mastectomy in women with a BRCA1 or BRCA2 mutation. N Engl J Med 2001;345:159-164.

12 Hartmann LC, Schaid DJ, Woods JE, et al.: Efficacy of bilateral prophylactic mastectomy in women with a family history of breast cancer. N Engl J Med 1999;340: 77-84.

13 Domchek SM, Friebel TM, Singer CF, et al.: Association of risk-reducing surgery in BRCA1 or BRCA2 mutation carriers with cancer risk and mortality. AMA 2010;304:967-975.
14 Rhiem K, Engel C, Graeser M, et al.: The risk of contralateral breast cancer in patients from BRCA1/2 negative high risk families as compared to patients from BRCA1 or BRCA2 positive families: a retrospective cohort study. Breast Cancer Res 2012;14:R156.

15 Biglia N, D’Alonzo M, Sgro LG, et al.: Breast cancer treatment in mutation carriers: surgical treatment. Minerva Ginecol 2016;68:548-556.

16 Valachis A, Nearchou AD, Lind P: Surgical management of breast cancer in BRCA-mutation carriers: A systematic review and meta-analysis. Breast Cancer Res Treat 2014;144:443-455.

17 van Sprundel TC, Schmidt MK, Rookus MA, et al.: Risk reduction of contralateral breast cancer and survival after contralateral prophylactic mastectomy in BRCA1 or BRCA2 mutation carriers. Br J Cancer 2005; 93:287-292.

18 Lee J, Park HY, Jung JH, et al.: Lymph node status as a prognostic factor in BRCA-positive breast cancer. J Surg Res 2017;215:125-131

19 Metcalfe K, Gershman S, Lynch HT, et al.: Predictors of contralateral breast cancer in BRCA1 and BRCA2 mutation carriers. Br J Cancer 2011;104:1384-1392.

20 Heemskerk-Gerritsen BA, Rookus MA, Aalfs CM, et al.: Improved overall survival after contralateral riskreducing mastectomy in BRCA1/2 mutation carriers with a history of unilateral breast cancer: a prospective analysis. Int J Cancer 2015;136:668-677.

21 Metcalfe K, Gershman S, Ghadirian P, et al.: Contralateral mastectomy and survival after breast cancer in carriers of BRCA1 and BRCA2 mutations: Retrospective analysis. BMJ 2014;348:g226.

22 Nava MB, Catanuto G, Pennati A, et al.: Conservative mastectomies. Aesthetic Plast Surg 2009;33:681-686.

23 Katerinaki E, Sircar T, Fatah F: Pre-expansion before risk reducing mastectomy combined with lipomodelling to enhance results from implant based reconstruction. J Plast Reconstruct Aesthet Surg 2012;65:182186.

24 Tondu T, Thiessen F, Tjalma WA: Prophylactic bilateral nipple-sparing mastectomy and a staged breast reconstruction technique: Preliminary Results. Breast Cancer (Auckl) 2016;10:185-189.
25 Dossett LA, Lowe J, Sun W, et al: Prospective evaluation of skin and nipple-areola sensation and patien satisfaction after nipple-sparing mastectomy. Surg Oncol 2016;114:11-16.

26 Gerber B, Marx M, Untch M, Faridi A: Breast reconstruction following cancer treatment. Dtsch Arzteb Int 2015;112:593-600; erratum Dtsch Arztebl Int 2016; 113:286.

27 Nestle-Krämling C, Bölke E, Budach W, Andree C Breast reconstruction after neoadjuvant radio chemotherapy: Review and personal technique IDEAL concept REV-EJMR-D-15-00268. Eur J Med Res 2016;21:

28 Frost MH, Schaid DJ, Sellers TA, et al.: Long term satisfaction and psychological and social function following bilateral prophylactic mastectomy. JAMA 2000; 284:319-324.

29 Zendejas B, Moriarty JP, O’Bryne J: Cost-effectiveness of contralateral prophylactic mastectomy versus routine surveillance in patient with unilateral breast cancer. J Clin Oncol 2011;29:2993-3000.

30 Schrauder MG, Brunel-Geuder L, Häberle L, et al. Cost-effectiveness of risk-reducing surgeries in preventing hereditary breast and ovarian cancer. Breast 2017;32:186-191.

31 Müller D, Danner M, Rhiem K, et al.: Cost-effectiveness of different strategies to prevent breast and ovarian cancer in German women with a BRCA 1 or $2 \mathrm{mu}$ tation. Eur J Health Econ. 2017 Apr 5. doi: 10.1007/ s10198-017-0887-5 [Epub ahead of print]

32 Hoskins PJ, Gotlieb WH: Missed therapeutic and prevention opportunities in women with BRCA-mutated epithelial ovarian cancer and their families due to low referral rates for genetic counseling and BRCA testing: A review of the literature. CA Cancer J Clin 2017;67: 493-506.

33 AGO Breast Committee: Diagnosis and treatment of patients with primary and metastatic breast cancer. Recommendations 2017. www.ago-online.de. 